01;05

\title{
Диссипация механической энергии в осциллирующем адгезионном контакте между жестким индентором и эластомером
}

\author{
(ㄱ Я.А. Ляшенко ${ }^{1,2}$, В.Л. Попов $1,3,4$ \\ ${ }^{1}$ Берлинский технический университет, Берлин, Германия \\ ${ }^{2}$ Сумский государственный университет, Сумы, Украина \\ ${ }^{3}$ Национальный исследовательский Томский государственный университет, Томск, Россия \\ ${ }^{4}$ Институт фозики прочности и материаловедения, Томск, Россия \\ E-mail: i.liashenko@tu-berlin.de
}

Поступило в Редакцию 22 июня 2020г.

В окончательной редакции 24 июля 2020 г.

Принято к публикации 28 июля 2020 г.

\begin{abstract}
Предложена модель гистерезиса в адгезионном контакте под действием осциллирующей нагрузки, возникающего из-за химической неоднородности поверхности индентора. Результаты численного моделирования сравниваются с экспериментальными данными, полученными на установке, позволяющей непосредственное наблюдение динамики области контакта. Показано, что гистерезис практически исчезает при амплитудах периодической нагрузки меньше критического значения, зависящего от максимальной глубины вдавливания.
\end{abstract}

Ключевые слова: адгезия, теория JKR, поверхностная энергия, гистерезис, эксперимент.

DOI: 10.21883/PJTF.2020.21.50197.18431

Адгезия играет большую роль в технике, медицине и живой природе $[1,2]$. Несмотря на большое количество исследований, до сих пор остается невыясненным механизм гистерезиса в адгезионных контактах. Если произвести индентирование на глубину $d$ и поменять направление движения, то практически всегда наблюдается гистерезис силы $[3,4]$. Этот факт противоречит классическим теориям, основанным на принципе энергетического баланса [5]. В настоящей работе показано, что неоднородность поверхностной энергии (обусловленная химической неоднородностью поверхностей) приводит к гистерезису, а тем самым и к потерям энергии в осциллирующих адгезионных контактах.

Рассмотрим контакт аксиально-симметричного жесткого индентора и упругого полупространства с модулем упругости $E$ и коэффициентом Пуассона $v$. Аксиальная симметрия позволяет использовать для решения контактной задачи метод редукции размерности (MDR) [6]. В этом методе трехмерный профиль индентора $f(r)$ замещается его одномерным эквивалентом $g(x)$ согласно правилу (рис. 1)

$$
g(x)=|x| \int_{0}^{|x|} \frac{f^{\prime}(r)}{\sqrt{x^{2}-r^{2}}} \mathrm{~d} r .
$$

Упругое полупространство заменяется массивом невзаимодействующих пружин с жесткостью $\Delta k=E^{*} \Delta x$, где $\Delta x-$ шаг дискретизации пространства, $E^{*}=E /\left(1-v^{2}\right)-$ эффективный упругий модуль. Для параболического индентора $f(r)=r^{2} /(2 R)$ имеем $g(x)=x^{2} / R$. При расчете контакта производится вдавливание профиля $g(x)$ в массив пружин (рис. 1) на глубину $d$, при этом сжатие каждой пружины равно $u(x)=d-g(x)$. При наличии адгезии пружины на границе контакта подтягиваются к индентору, увеличивая радиус контакта $a$, который определяется по правилу Гесса [6]:

$$
\Delta l_{\max }=-\sqrt{2 \pi a \Delta \gamma / E^{*}},
$$

где $\Delta l_{\max } \equiv u(a)$ - натяжение крайних пружин, $\Delta \gamma-$ удельная работа адгезии, которая в химически неоднородной системе сама может зависеть от радиуса контакта. С учетом (2) глубина индентирования $d$ запишется в виде [6]:

$$
d=a^{2} / R-\sqrt{2 \pi a \Delta \gamma / E^{*}} .
$$

Действующая в контакте нормальная сила - это сумма сил от всех пружин [6]:

$$
\begin{aligned}
F(a) & =E^{*} \int_{-a}^{a} u(x) \mathrm{d} x=2 E^{*} \int_{0}^{a}\left(d-x^{2} / R\right) \mathrm{d} x \\
& =4 E^{*} a^{3} /(3 R)-\sqrt{8 \pi a^{3} E^{*} \Delta \gamma} .
\end{aligned}
$$

Выражения (3) и (4) представляют классический результат теории JKR (Johnson-Kendall-Roberts) [5] и задают зависимость контактной силы от глубины индентирования $F(d)$.

В [7] изучалась ситуация, когда поверхность индентора химически неоднородна и представляет собой чередование полос равной толщины с различными значениями поверхностной энергии $\Delta \gamma$. В реальности распределения поверхностной энергии, как правило, более сложные. В строгом смысле для описания трехмерного контакта c неоднородной поверхностной энергией метод редукции размерности неприменим, поскольку контакт теряет 

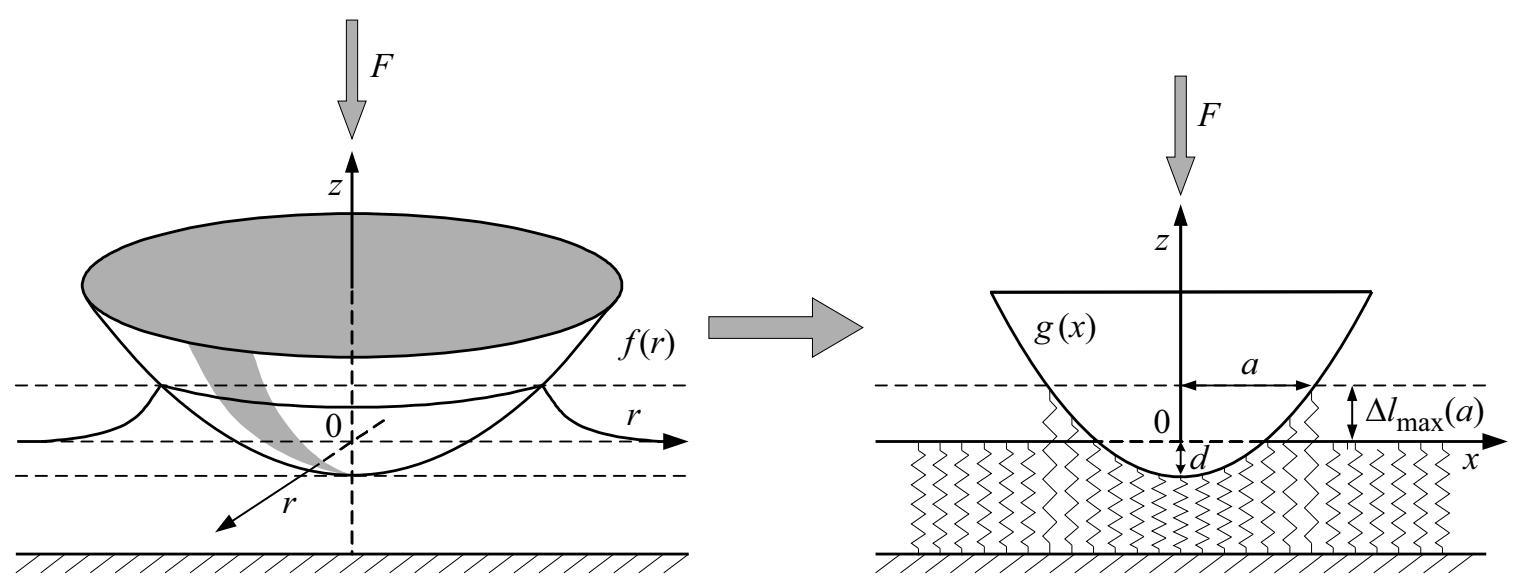

Рис. 1. Графическая визуализация метода редукции размерности.

аксиальную симметрию. Неоднородность поверхностной энергии, однако, сравнительно слабо влияет на форму контакта, поскольку последняя определяется нелокальными упругими взаимодействиями. Если на поверхности индентора выделить относительно центра контакта кольца малой толщины $h$ с радиусами $a_{i}(x)$, то усредненную по каждому кольцу энергию трехмерной поверхности $\Delta \gamma(x)$ можно использовать для определения радиуса контакта $a$ по правилу (2). Полученное таким образом решение будет приближенным решением трехмерной задачи в том случае, когда контакт близок к симметричному, что часто наблюдается как для химически неоднородных, так и для шероховатых поверхностей $[8,9]$.

В численной процедуре при погружении профиля $g(x)$ в упругое основание контактирующие пружины определяются по правилу (2), а действующая сила рассчитывается как сумма сил от всех пружин. Вид распределения $\Delta \gamma(x)$ оказывает существенное влияние на результат [7,8]. Распределение $\Delta \gamma(x)$ может иметь различный вид, поскольку оно формируется в результате сложных физико-химических процессов, включающих окисление, коррозию, износ, загрязнение и т.п. Мы остановимся на качественном описании неоднородности, не рассматривая причины возникновения точного распределения $\Delta \gamma(x)$, что может служить темой отдельного исследования. Для формирования случайного распределения $\Delta \gamma(x)$ будем использовать функцию Бокса-Мюллера $\Delta \gamma=2\left(-I \ln k_{1}\right)^{1 / 2} \cos \left(2 \pi k_{2}\right)$ [10] с дисперсией $\sigma^{2}=2 I$. Случайные числа с равномерным распределением $k_{1}$ и $k_{2}$ принадлежат диапазону $(0,1]$. При расчете $\Delta \gamma(x)$ будем выбирать только те сгенерированные значения $\Delta \gamma$, которые находятся в интервале $0.05<\Delta \gamma<0.3 \mathrm{~J} / \mathrm{m}^{2}$, поскольку поверхностная энергия всегда имеет минимальное и максимальное значения, во многом определяющие поведение системы [7]. Результаты моделирования показаны на рис. 2, где кривая 1 соответствует универсальной зависимости $F(d)$, по которой происходит индентирование, а кривая 2 универсальной зависимости на стадии отрыва. Рассмот- рим подробно процесс индентирования. Точка $A-$ это точка первого контакта при $d=0$. После возникновения контакта он быстро распространяется до равновесного значения (точка $B$ ), а сила $F$ становится отрицательной. На участке $B C$ происходит индентирование до значения $d_{\max }=0.05 \mathrm{~mm}$. В точке $C$ индентор начинает движение в обратную сторону. При этом на участке $C D$ площадь контакта сохраняется постоянной, а зависимость $F(d)$ линейна. После перехода на показанную кривой 2 зависимость контакт начинает уменьшаться до полного разрушения по пути $D H E$. На этом заканчивается полный цикл индентирования $A B C D H E$. Второй численный эксперимент - это цикл $A B C F G D H E$, соответствующий большему значению $d_{\max }=0.15 \mathrm{~mm}$. Здесь индентирование также происходит по кривой 1 , а разрушение контакта описывается зависимостью 2, на участке $F G$ площадь контакта постоянна. В обоих показанных циклах индентирования осуществляется диссипация механической энергии, описываемая площадью замкнутой фигуры $F(d)$, соответствующей полному циклу индентирование/отрыв. В случае $d_{\max }=0.15 \mathrm{~mm}$ это фигура $A B C F G D H E A$. В [3] экспериментально обнаружено, что диссипация энергии может быть разделена на две составляющие: „не зависящую“ и „зависящую“ от глубины индентирования $d_{\max }$. На рис. 2 не зависящим от глубины индентирования $d$ для обоих численных экспериментов является участок $D H E$, поскольку после перехода на кривую 2 отрыв индентора описывается общей зависимостью $F(d)$. Но если изначально произвести индентирование на глубину $d_{\max }<0.05 \mathrm{~mm}$, то „независимый“ участок станет короче, поскольку при отрыве будет реализоваться меньшее значение площади контакта $A$, а контакт будет обладать меньшей жесткостью. Если после точки первого касания $A$ и распространения контакта до равновесного значения (точка $B$ ) произвести отрыв индентора и при этом система выйдет на кривую 2 (такой переход показан штриховой линией $B H$ ), то при дальнейшем уменьшении $d$ действительно наблюдается универсальный „независимый“ участок $F(d)$. На 

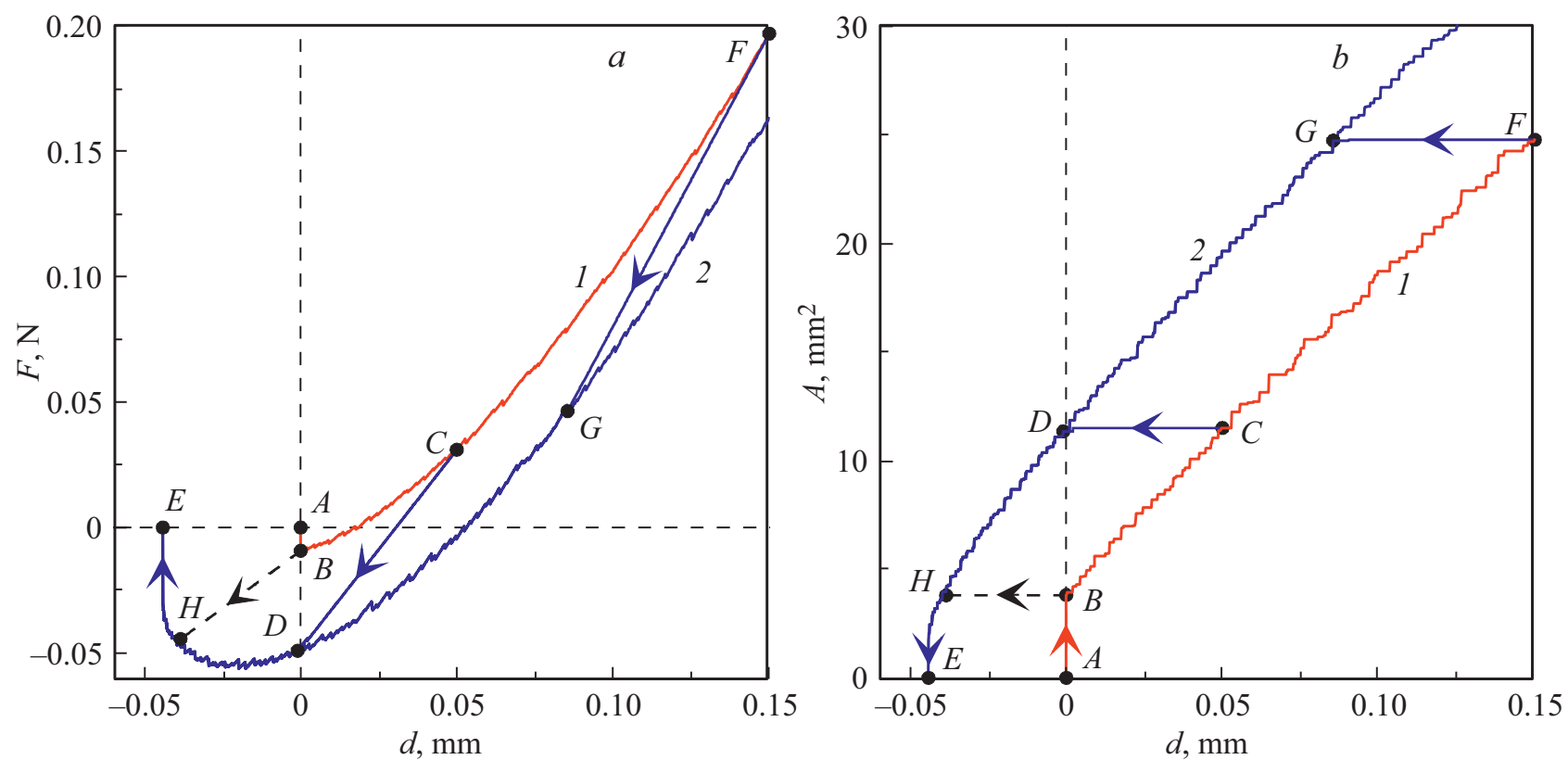

Рис. 2. Зависимости контактной силы $F(a)$ и площади контакта $A(b)$ от глубины индентирования $d$ при параметрах $R=40$ mm, $E=0.324 \mathrm{MPa}, v=0.47, I=1 \mathrm{~J}^{2} / \mathrm{m}^{4}, 0.05<\Delta \gamma<0.3 \mathrm{~J} / \mathrm{m}^{2}$. Параметры, при которых построен рисунок, соответствуют параметрам реального эксперимента $[8]$. Видео доступно в онлайн-версии статьи.
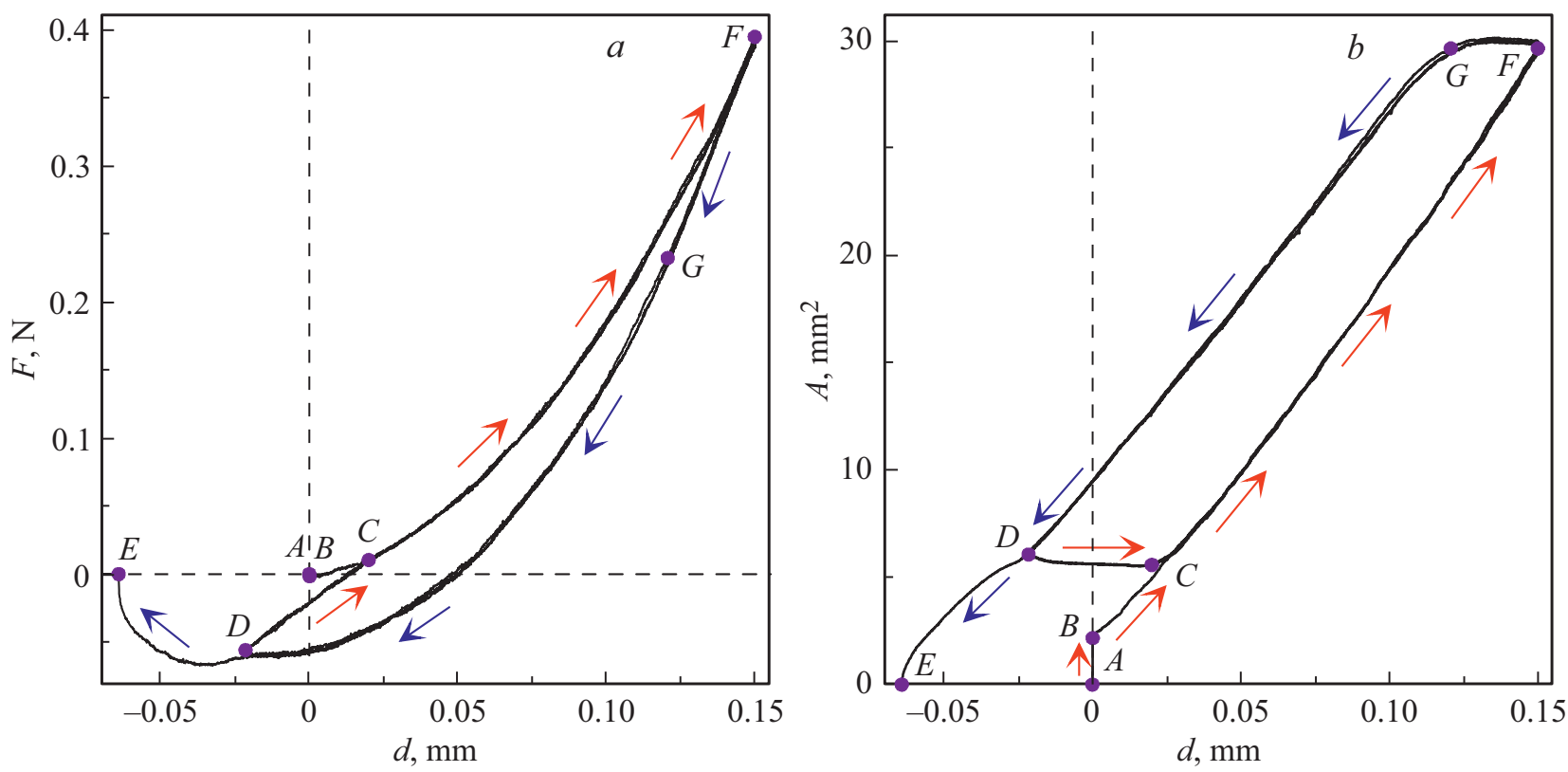

Рис. 3. Экспериментально измеренные зависимости контактной силы $F(a)$ и площади контакта $A(b)$ от глубины индентирования $d$ при индентировании сферического стального индентора с радиусом $R=40 \mathrm{~mm}$ в лист оптически прозрачной резины TARNAC CRG N3005 толщиной $h=5 \mathrm{~mm}$ с упругими параметрами $E=0.324 \mathrm{MPa} \mathrm{и} v=0.47$. Видео доступно в онлайн-версии статьи.

отрезке $B H$ площадь контакта постоянна, после чего на участке $H E$ она быстро уменьшается и контакт разрушается. Участок зависимости $F(d)$, на котором диссипация энергии не зависит от глубины индентирования, - это короткий участок $H E$, находящийся близко к точке потери стабильности и разрушения контакта $E$. На отрезках $C D$ и $F G$ на рис. 2 радиус контакта и контактная жесткость не изменяются, поэтому при осциллирующем движении вдоль линейных участков такого типа гистерезис и диссипация энергии отсутствуют. Но если после прохождения пути $A B C F G D$ сразу изменить направление движения на противоположное, то дальнейшее индентирование пойдет по участку $D C F$, т. е. при циклическом движении между точками $d=d_{F}$ и $d=d_{D}$ (значения $d_{F}$ и $d_{D}$ отвечают точкам $F$ и $D$ на рис. 2) происходит движение по замкнутой траек- 
тории $C F G D C$, а диссипация энергии описывается ее площадью на зависимости $F(d)$. Изначально система выходит на указанный цикл по пути $A B C F$.

Для верификации предложенной модели был проведен эксперимент по методике и с помощью установки, которые подробно описаны в $[8,9]$. Проводилось индентирование стального сферического индентора радиусом $R=40 \mathrm{~mm}$ в лист прозрачной резины TARNAC CRG $\mathrm{N} 3005$ с толщиной $h=5 \mathrm{~mm}$, который находился на стеклянной подложке. Для исключения вязкоупругих эффектов индентор сдвигался с очень низкой скоростью $v=0.5 \mu \mathrm{m} / \mathrm{s}$. В работе [11] было экспериментально продемонстрировано, что для рассматриваемой системы вязкоупругие свойства начинают проявляться при значительно более высоких скоростях $v>16 \mu \mathrm{m} / \mathrm{s}$. Перед проведением эксперимента поверхность зеркально отполированного индентора была обработана для создания шероховатости небольшой амплитуды. Это было сделано для того, чтобы индентор диффузно рассеивал освещение и контакт отчетливо выделялся на фотографиях контактной области. Измеренные параметры шероховатости поверхности индентора следующие: среднеквадратичная шероховатость $h_{\mathrm{RMS}}=4.48 \mu \mathrm{m}$, среднеквадратичный наклон $\kappa=5.18$ (подробно о методике измерения шероховатости см. в [9]). Результаты эксперимента показаны на рис. 3, на котором обозначены точки, соответствующие точкам на рис. 2. Здесь сначала осуществлялось индентирование на глубину $d=0.15 \mathrm{~mm}$, затем индентор вытягивался до координаты $d=-0.025 \mathrm{~mm}$, после чего снова сдвигался к уровню $d=0.15 \mathrm{~mm}$, и так несколько раз. На рис. 3 траектории $C F$ и $F G D$ представлены тремя кривыми, поскольку индентор проходил по ним три раза, а отрезок $D C$ состоит из двух наложенных кривых. Исходя из совпадения зависимостей можно констатировать хорошую повторяемость эксперимента. В эксперименте на стадии индентирования точки $A$ и $B$ на зависимости $F(d)$ практически совпадают (рис. $3, a$ ), поскольку контакт при первом касании распространяется незначительно $[8,9]$. При разрушении контакта на участке $D E$ на зависимости $F(d)$ реализуется дополнительный минимум, поскольку при циклическом движении индентора центральный участок контакта (значение площади в точке $D$ на рис. $3, b)$ находится все время в контакте и за счет этого успевает упрочниться [8]. Этот эффект отсутствует на теоретических кривых, поскольку при моделировании эффект старения контакта не учитывался. В заключение заметим, что химическая неоднородность является только одним из механизмов гистерезиса в адгезионных контактах. Аналогичное поведение может быть следствием шероховатости контактирующих поверхностей $[9,12]$.

\section{Финансирование работы}

Работа выполнена при финансовой поддержке Немецкого научно-исследовательского сообщества (DFG) (проект РО 810-55-1) и государственного задания ИФПМ СО РАН (проект III.23.1.1).

\section{Конфликт интересов}

Авторы заявляют, что у них нет конфликта интересов.

\section{Список литературы}

[1] Gorb $S$. Adhesion and friction in biological systems. Springer, 2012. 280 p.

[2] Kendall K. Molecular adhesion and its applications. The Sticky Universe. Kluwer Academic Publ., 2001. 429 p. DOI: $10.1007 / \mathrm{b} 100328$

[3] Deng W., Kesari H. // Sci. Rep. 2019. V. 9. P. 1639. DOI: $10.1038 / \mathrm{s} 41598-018-38212-\mathrm{z}$

[4] Dalvi S., Gujrati A., Khanal S.R., Pastewka L., Dhinojwala A., Jacobs T.D.B. // Proc. Natl. Acad. Sci. USA. 2019. V. 116. P. 25484-25490. DOI: 10.1073/pnas.1913126116

[5] Johnson K.L., Kendall K., Roberts A.D. // Proc. Roy. Soc. Lond. A. 1971. V. 324. P. 301-313. DOI: $10.1098 /$ rspa.1971.0141

[6] Popov V.L., Heß M., Willert E. Handbook of contact mechanics: exact solutions of axisymmetric contact problems. Berlin: Springer, 2019. 347 p. DOI: 10.1007/978-3-662-58709-6

[7] Popov V.L. // Preprints. 2020. 2020030131. P. 1. DOI: $10.20944 /$ preprints202003.0131.v1

[8] Ляменко Я.А., Попов В.Л. // ЖТФ. 2020. Т. 90. В. 10. C. 1769-1782. DOI: 10.21883/JTF.2020.10.49812.57-20

[9] Lyashenko I.A., Pohrt R. // Front. Mech. Eng. 2020. V. 6. P. 49. DOI: $10.3389 /$ fmech.2020.00049

[10] Box G.E.P., Muller M.E. // Ann. Math. Statist. 1958. V. 29. P. 610-611. DOI: $10.1214 / \mathrm{aoms} / 1177706645$

[11] Lyashenko I.A., Popov V.L. // AIP Conf. Proc. 2019. V. 2167. P. 020201. DOI: 10.1063/1.5132068

[12] Popov V.L. // AIP Conf. Proc. 2019. V. 2167. P. 020286. DOI: $10.1063 / 1.5132153$ 